\title{
Stability of Nonlinear Descriptor Systems and Applications to Stabilization of Quadcopters
}

\author{
Drilon Bunjaku ${ }^{1}$, Jovan D. Stefanovski ${ }^{2}$, Georgi Dimirovski ${ }^{3}$ \\ ${ }^{1}$ Faculty of Electrical and Computer Engineering at University of Prishtina, Kodra \\ e Diellit p.n., 10000 Prishtina, Republic of Kosovo, drilon.bunjaku@uni-pr.edu \\ ${ }^{2}$ Control \& Informatics Div. JP "Strezevo", Boulevard 1st of May b. b., 7000 \\ Bitola, R. N. Macedonia, jovanstef@t.mk \\ ${ }^{3}$ Dogus University Acibadem-Kadikoy, 34722 Istanbul, Republic of Turkey, \\ gdimirovski@dogus.edu.tr
}

\begin{abstract}
In this paper, new sufficient Lyapunov-like stability conditions and new Lyapunov-like, stabilizability conditions for nonlinear descriptor systems, with control, are presented along with their applications to quadcopter's flight stabilization. Most of these results are rather practical because they do not need solving nonlinear differential equations and nonlinear algebraic equations. Thus, the usage of these new results requires only the differentiation of functions. The numerical simulation results prove that the stabilization of the quadcopter is improved, in comparison with pure tracking control.
\end{abstract}

Keywords: Nonlinear descriptor system; Lyapunov-like stability; Nonlinear hybrid control; Quadcopter stabilization

\section{Introduction}

Considerable new developments appeared over the last couple of decades, within the literature, on various hybrid dynamical systems [1], [2]. Certain segments of these developments are called differential-algebraic systems while others hybrid systems, one subclass of which obeys switching law in terms of algebraic part hence known as switched systems, while another subclass is referred to as nonlinear descriptor systems and is of main concern in this study. Nonetheless, the word is about an equivalent case of nonlinear dynamical continuous-time systems to linear descriptor systems. These can be best envisaged by the original system created in 1951 by A. I. Lurye [3] at the Control Science Institute of Academy of Sciences in Moscow. Namely, Lurye's nonlinear systems had a feedback architecture with one static sector nonlinearity and a linear dynamic component, classical one, which could be conceptualized as descriptor, linear as in Figure 1. 
The herein considered, nonlinear descriptor systems can be readily envisaged as a generalization of Lurye by assuming the linear part is described by descriptor state-variable and output equation as depicted in Fig. 1. Obviously, this figure highlights the underlying control physics of nonlinear descriptor systems. Feedback systems having such an architecture became known as Lurye-type of systems (also called nonlinear control systems with separable nonlinearity) while their stability as the Lurye stability problem. Ultimately LaSalle and Lefschetz solved it in [4], by proving that Lurye's candidate Lyapunov function was indeed a proper Lyapunov function, for his class of nonlinear systems [3]. Many years later, in 2000, the essential fundamental result on the stability of multivariable Lurye systems [5] appeared.

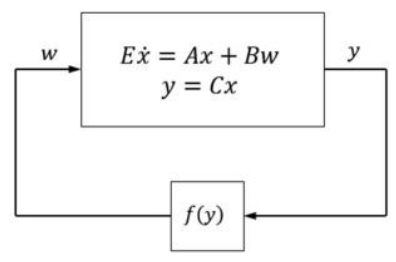

Figure 1

Lurye-type of nonlinear descriptor systems

To date, the most thorough recent study for Lurye-type subclass of nonlinear descriptor systems was contributed by M. Ikeda and collaborators of Graduate Scholl of Engineering his at Osaka University; see [6]-[8] and references therein. In 2004, they have derived absolute stability criteria for Lurye systems with asymmetric nonlinearities [6], and in 2006 they published stability theory for descriptor system with non-smooth nonlinearities [7]. Furthermore, in 2008, they solved the absolute stability problem for multivariable Lurye-type descriptor systems [8]. Thus, we consider, nowadays, the concept of nonlinear descriptor systems is well established and their mathematical description generalized to differential-algebraic equations like (1) and (2) presented below.

In the sequel, the following combined differential and algebraic equations define the descriptor nonlinear systems of interest in this study. Consider the system of differential and algebraic equations (descriptor system)

$$
\begin{aligned}
& \dot{x}_{1}(t)=\boldsymbol{f}\left(x_{1}(t), x_{2}(t)\right), \\
& 0=\boldsymbol{h}\left(\mathrm{x}_{1}(\mathrm{t}), \mathrm{x}_{2}(\mathrm{t})\right),
\end{aligned}
$$

where $x_{1}=x_{1}(t) \in \mathbf{N}_{1} \subseteq \mathbf{R}^{n_{1}}, \quad x_{2}=x_{2}(t) \in \mathbf{N}_{2} \subseteq \mathbf{R}^{n_{2}}$.

Let denote $x=x(t):=\left[x_{1}(t) x_{2}(t)\right]^{T} \in \mathbf{N}=\mathbf{N}_{1} \times \mathbf{N}_{2} \subseteq \mathbf{R}^{n}, n=n_{1}+n_{2}$. Let denote by $\left[\begin{array}{ll}x_{10} & x_{20}\end{array}\right]^{T}=x_{0}$ the initial condition for the descriptor system (1) and (2). 
The function $\boldsymbol{f}\left(x_{1}, x_{2}\right)$ and $\boldsymbol{h}\left(x_{1}, x_{2}\right)$ are assumed to have partial derivatives of all orders in $\mathbf{N}$. Even though, the existence of all partial derivatives is not needed in all of our results.

The set $\mathbf{N}$ must be invariant with respect to the dynamics (1) and (2), i.e. once $x_{0} \in \mathbf{N}$ we must have $x(t) \in \mathbf{N}$ for all $t>0$. The importance of the sets $\mathbf{N}$ is illustrated in Example 3. Obtaining the set $\mathbf{N}$ is not easy if there is no physical interpretation of the mathematical model (1) and (2). In that case, estimation mathematical techniques could be applied, as in Example 4 of [4] (pages 68-70).

Assumption 1: $\boldsymbol{f}(0,0)=0$ and $\boldsymbol{h}(0,0)=0$

Notice that this assumption is a natural consequence of the fundamental laws of physics.

The systems of the form (1) and (2) became of considerable current interest nowadays; see [2] and references therein. Nonetheless, there are three reasons at least why we consider such systems:

i. If the system includes both linearities and nonlinearities, then it is convenient if we can separate the nonlinearities into algebraic equations of the type $\boldsymbol{h}\left(x_{1}, x_{2}\right)=0$. (Obviously, then $\boldsymbol{f}\left(x_{1}, x_{2}\right)$ is a linear dependence of the form $\boldsymbol{f}\left(x_{1}, x_{2}\right)=A_{1} x_{1}+A_{2} x_{2}$ for some constant matrices $A_{1}$ and $\left.A_{2}\right)$. For example, in electrical circuits containing nonlinear elements (for instance, diodes) the nonlinear block $\boldsymbol{h}(x)=0$ can be chosen so that it contains the nonlinear elements only.

ii. In many systems, a passive [9] or made passive by feedback [10]-[12] (usually referred to as 'passified'), or else conservative [13] some part could appear separated.

It is important to mention works [14], [15] and later [16] which marked the beginning of studies in dissipative and passive dynamical systems (the linear ones, in particular), as well as, passivity-oriented reliable control developments.

iii. The holonomic constraints in mechatronic systems are modelled by the algebraic constraints of the type (2) (see [17]). As we shall show in Section 3.1 the descriptor system (1) and (2) is a natural model for the typical quadcopter construction.

In order to motivate our concept, we discuss here the drawbacks of the two wellaccepted concepts for stabilization of affine-in-control nonlinear systems that are given by:

$\dot{x}=\boldsymbol{f}(x)+\boldsymbol{G}(x) u, x \in \mathbf{R}^{n}, \quad u \in \mathbf{R}^{m}$, 


$$
y=h(x)+E(x) u, \quad y \in \mathbf{R}^{m},
$$

where $u$ is the control variable and $y$ is the output variable. Namely, these are the concept of feedback passivization [12], [19], [37] (see Section 2.4 in [37]) and the concept of control Lyapunov functions (see Section 3.2 in [37]). The first concept requires the existence of a function $v(x)$ such that:

$$
\frac{\partial \boldsymbol{v}(x)}{\partial x} \boldsymbol{g}_{i}(x)=\boldsymbol{h}_{i}(x), \quad i=1,2, \ldots, m
$$

where $\left[\boldsymbol{g}_{1}, \boldsymbol{g}_{2}, \ldots, \boldsymbol{g}_{m}\right]:=\boldsymbol{G}$ and $\left[\boldsymbol{h}_{1}, \boldsymbol{h}_{2}, \ldots, \boldsymbol{h}_{m}\right]^{\mathrm{T}}:=\boldsymbol{h}$. In the case of vector control $(m>1)$, the system of partial differential equations (5) has no solution in the generic case of controllable system. (Also see the Comment (3) on page 1225 in [13]). Therefore, the concept of feedback passivization does not use the controllability property in a positive way.

In the concept of control Lyapunov functions, one has to find a so-called control Lyapunov function $\mathbf{v}(x)$ such that the following implication relationships hold:

$$
\frac{\partial \boldsymbol{v}(x)}{\partial x} \boldsymbol{g}_{i}(x)=0, i=1,2, \ldots, m \Rightarrow \frac{\partial \boldsymbol{v}(x)}{\partial x} \boldsymbol{f}(x)=0 .
$$

Apart of the fact that it is more involved to find a control Lyapunov function than to find a Lyapunov function, in order to check the validity of implication (6), the system on the left in (6) has to be solved in $\boldsymbol{v}$. However, analogous to the case of system (5), this system too has no solution in the generic case.

The contributions of this paper are:

Theorem 1, which is one of the main results of this paper, can be regarded as a generalization of the second part of the Corollary 1 in [18] (i.e., going from linear descriptor systems to nonlinear descriptor systems). Theorem 2, which is also one of the main results in here, does generalize Theorem 4 in [18] (i.e., going from controlled systems to controlled descriptor systems). Also, the four corollaries in this paper guarantee stability and stabilizability as these Theorems 1 and 2 do, respectively. But our conditions, which are based on differentiation of functions, actually do represent observability and controllability; besides, they are more useful in practical applications. In particular, Corollary 4 uses the controllability rather in a positive way.

Theorem 2, is advanced, in this the paper, by its application to the quadcopter flight stabilization problem. In particular:

i. We consider a positive semidefinite Lyapunov-like function, instead of positive definite one, for the reason that the moving of quadcopter in the horizontal direction, in general, requires that at least one of the coordinates does not approach zero when $t \rightarrow \infty$. Precisely, if we denote by $x(t)$ the 
position of the quadcopter in the moving direction, then we require $x(t)$ to be a ramp signal (then the corresponding velocity is nonzero), instead of $x(t) \rightarrow \infty$ when $t \rightarrow \infty$. In addition, the motion is possible only if $\theta \neq 0$ or $\phi \neq 0$, where $\theta$ and $\phi$ are quad rotor angles (attitude angles). Therefore, the coordinate $x$, as well as the attitude coordinates, are not allowed directly in the Lyapunov-like function.

ii. We consider a descriptor system model of the quadcopter for the reason that there is no analytic inversion of the algebraic dependency (see also Remark 1 and the algebraic dependences (27)).

In the applications-oriented research towards electrical power systems, the actual novel contribution is to the so-called coordinated passivation control of nonlinear systems. It was first introduced by work [19] and further extended in [20]-[25]. The works [21]-[25] have expended the ideas of dissipativity and passivity further to control designs for switched dynamical systems.

Quadcopters are known for their highly nonlinear motion dynamics and so nonlinear models are indispensable. A common practical procedure is to linearize the nonlinear quadcopter dynamic model around a hovering mode (the operating steady-state) since, due to its symmetric frame, the linear dynamic model becomes somewhat simplified, and therefore, it is easier to design linear controllers; see ([26-30], [39]). A drawback of such linearized model, however, is the operation in the immediate proximity of the linearizing state point (hovering mode) must be secured. Once the quadcopter is out of this proximity region, the system becomes uncontrollable using linear control strategies, hence, it is highly possible that quadcopter will crash. Often, quadcopters are called under-actuated physical systems, where the translational motion in $x-y$ plane are performed through the rotational motions $\theta, \phi$. Namely, the 6-DoF flying object could not be controlled with a single loop, and thus, a cascade control system is indispensable. In order to recover and keep vicinity of the aforementioned hovering mode, there are various control approaches developed under the cascade control concept, where the inner loop ought to be designed at least 5 times faster than the outer loop; see [27]-[30], [39].

In this paper, we elaborate a new approach with respect to: (a) representing a quadcopter model as the descriptor system, i.e. system with Differential-Algebraic Equations; (b) designing a nonlinear control. For, recently it was shown in [31] and [40], that the class of descriptor state-vector systems appears more advanced in describing the nature of physical systems than ordinary state-vector systems. It should be noted that, the descriptor system approach has been already applied successfully to aircraft control design [32] (see Example 7.2 in there).

Remarks on the notation. The scalars and vector are denoted by lower-case letters, while the matrices are denoted by upper-case letters. The functions of the 
state variable $x$ are denoted by bold-faced letters, and if it is not ambiguous, we omit the dependence on $x$ frequently. If $v$, is a scalar function, the notation $\frac{\partial v}{\partial x}$ means $\left[\frac{\partial v}{\partial x_{1}}, \ldots, \frac{\partial v}{\partial x_{n}}\right]$ where $x_{1}, \ldots, x_{n}$ are the entries of the vector-column $x$.

\section{Main Results}

In this section, the following assumption specifies the specific mathematical preliminaries that are required in this study.

\subsection{On the Stability of Nonlinear Descriptor Systems}

The following assumption specifies certain mathematical preliminaries that are needed in this study.

Assumption 2:

(I) The vector function $\boldsymbol{h}$ has $n_{2}$ entries, and the algebraic equation (2) defined in $\mathbf{N}$ is equivalent with the equation $x_{2}=\gamma\left(x_{1}\right)$, for some vector function $\gamma(\cdot)$.

(II) The system given by (1) and (2) has unique finite solution $x(t)$ for all $t>0$ and all initial conditions $x(0)=x_{0}$.

Remark 1. (i) Assumption 2 (I) is a necessary condition for uniqueness of the solution of the descriptor system (1) and (2).

(ii) By replacement of the dependency $x_{2}=\gamma\left(x_{1}\right)$ in the formula (1), we obtain a system of ordinary differential equations $\dot{x}_{1}=f\left(x_{1}, \gamma\left(x_{1}\right)\right)$, on which the standard existence and uniqueness results can be applied (for instance, Existence Theorem on page 23 in [4]). The reason why we consider the descriptor system, and not the ordinary system $\dot{x}_{1}=f\left(x_{1}, \gamma\left(x_{1}\right)\right)$ is that the explicit dependency $x_{2}=\gamma\left(x_{1}\right)$ cannot be found in general.

(ii) Note that under Assumption 2, impulsive solutions at $t=0$ of the descriptor system (1) and (2) cannot appear. Only finite jumps can appear at $t=0$, if the initial conditions are not consistent [12].

Remark 2. Assumption 2 is not unrealistic. Indeed, sufficient conditions for Assumption 2 for nonlinear discrete-time descriptor system 
$E x_{k+1}=A x_{k}+B \phi\left(k, x_{k}\right)$, where the pair $(E, A)$ is regular and causal, are given by (2) and (8) in [41]. The condition (2) is a Lipschitz-type condition on $\phi$, while the condition (8) guarantees, together with (2), the contractivity of a function, needed to apply the fixed-point theorem, instead of the implicit function theorem, used in some papers referenced in [41].

Remark 3. Assumption 2 is natural. Indeed, if the equations (1) and (2) model a real system, then if Assumption 2 is not satisfied, finite escape times can appear in the solution, which cannot appear in practice. In this case, we have to modify the model equations (1) and (2). However, the problem to establish that there are no finite escape times is not easy. It is connected with the problem of obtaining the set $\mathbf{N}$, for which we have already stated that it is not easy. (See Example 3 where we obtain the set $\mathbf{N}$ and checked the existence of finite escape times but, only for a very simple descriptor system.)

Finally, we could give our stability results without Assumption 2, like in Theorem VIII of seminal monograph [4], which restricts only on the bounded solutions for $0 \leq t<\infty$. Another possibility is to restrict $\mathbf{N}$ to be a bounded set, like in Theorem VI of [4]. The third possibility is to consider the local asymptotic stability property, i.e. all points $x_{0}$ in a neighborhood of the origin satisfy $x(0)=x_{0} \Rightarrow x(t) \rightarrow 0$ when $t \rightarrow \infty$.

An obvious consequence of Assumption 2 (I) is the following proposition.

Proposition 1: Matrix $\partial \boldsymbol{h} / \partial x_{2}$ is nonsingular in $\mathbf{N}$.

Theorem 1: Under Assumptions 1 and 2, if there exists a function $v\left(x_{1}\right)$ such that

(i) $\boldsymbol{v}\left(x_{1}\right) \geq 0$ for all $x_{1} \in \mathbf{N}_{1}$ and $\boldsymbol{v}(0)=0$,

(ii) $\boldsymbol{\varphi}\left(x_{1}, x_{2}\right):=\frac{\partial \boldsymbol{v}}{\partial x_{1}} \boldsymbol{f}\left(x_{1}, x_{2}\right)=\dot{v} \leq 0$ for all $x \in \mathbf{N}$ satisfying (2),

(iii) The solution $x(t)$ of the descriptor system given by

$\dot{x}_{1}=\boldsymbol{f}\left(x_{1}, x_{2}\right), \boldsymbol{h}\left(x_{1}, x_{2}\right)=0, \boldsymbol{\varphi}\left(x_{1}, x_{2}\right)=0$

satisfies $x(\infty)=\lim _{t \rightarrow \infty} x(t)=0$ for all initial conditions $x(0)=x_{0}$, then the solution $x(t)$ of the descriptor systems given by (1) and (2) satisfies $x(\infty)=\lim _{t \rightarrow \infty} x(t)=0$ for all initial conditions $x(0)=x_{0}$.

Proof. For some $a \geq 0$, define the point set $\mathbf{E}_{a}=\left\{x \in \mathbf{N}: 0 \leq \boldsymbol{v}\left(x_{1}\right) \leq a\right\}$. Under the condition (i) and (ii), the set $\mathbf{E}_{a}$ is invariant with respect to dynamics (1) and (2). Indeed, since $0 \leq \boldsymbol{v}\left(x_{1}(t)\right) \leq \boldsymbol{v}\left(x_{10}\right) \leq a$, we have $0 \leq \boldsymbol{v}\left(x_{1}(t)\right) \leq a$. Denote by $\mathbf{M}$ 
the point set $\mathbf{M}=\{x \in \mathbf{N}: \boldsymbol{\varphi}(x)=0\}$. By virtue of Theorem VI in [4], all solutions $x(t)$, starting with $x(0)=x_{0} \in \mathbf{N}$ must tend to the largest invariant set contained in $\mathbf{M}$.

Remark 4. That the absence of finite escape time in Theorem 1 is really necessary, we can see by Example 1.1 in [33], i.e.

$\dot{x}=-0.5(1+z) x^{3}, \dot{y}=z, \quad \dot{z}=-2 a z-a^{2} y$,

for some real number $a>0$. (The algebraic identities (2) are void.) The initial conditions are $x(0)=x_{0}, \mathrm{y}(0)=1$ and $\mathrm{z}(0)=0$. The analytical solution for $x(t)$ satisfies

$x(t)^{2}=x_{0}^{2} /\left\{1+x_{0}^{2}\left[t+(1+a t) e^{-a t}-1\right]\right\}$,

therefore, if $x_{0}^{2}>1$, a finite escape time appears for sufficiently large $a$. (This kind of dynamic event is called peaking phenomenon in [33].)

On the other hand, the conditions (i), (ii) and (iii) of Theorem 1 are satisfied with $\mathbf{v}=\left[\begin{array}{ll}y & z\end{array}\right]\left[\begin{array}{cc}\frac{5}{4 a}+\frac{a}{4} & \frac{1}{2 a^{2}} \\ \frac{1}{2 a^{2}} & \frac{1}{4 a^{3}}+\frac{1}{4 a}\end{array}\right]\left[\begin{array}{l}y \\ z\end{array}\right]$. Indeed, we have $\boldsymbol{v}>0$, for all $[y, z] \neq 0$, and $\dot{\boldsymbol{v}}=-\left[\begin{array}{ll}y & z\end{array}\right]\left[\begin{array}{ll}1 & 0 \\ 0 & 1\end{array}\right]\left[\begin{array}{l}y \\ z\end{array}\right]<0$, for all $[y, z] \neq 0$.

The condition $\dot{\boldsymbol{v}}=0$ implies that $y=0, z=0$ and $\dot{x}=-0.5 x^{3}$, whose solution $x(t) \rightarrow 0$ when $t \rightarrow \infty$.

Example 1. In work [34], the below stated result is proved as Theorem 1. Namely, consider the regular descriptor system

$$
E \dot{x}=A x,
$$

and suppose there is a solution $P$ of the following equations:

$E^{T} P=P^{T} E$,

$A^{T} P+P^{T} A+C^{T} C=0$,

for some matrix C. It is proved in [34], if the pair $(A-s E, C)$ is impulse observable and finite mode detectable, and if $E^{T} P=P^{T} E \geq 0$ holds, then the descriptor system (8) is asymptotically stable and impulse-free. 
Let us now prove this Theorem 1 of [34] by means of our Theorem 1. For this purpose, consider $\boldsymbol{f}\left(x_{1}, x_{2}\right)$ and $\boldsymbol{h}\left(x_{1}, x_{2}\right)$ are represented by linear dependencies

$\boldsymbol{f}\left(x_{1}, x_{2}\right)=A_{11} x_{1}+A_{12} x_{2}$ and $\boldsymbol{h}\left(x_{1}, x_{2}\right)=A_{21} x_{1}+A_{22} x_{2}$, for some matrices $A_{11}, A_{12}, A_{21}$ and $A_{22}$. Further, denote

$E=\left[\begin{array}{cc}I_{n_{1}} & 0 \\ 0 & 0\end{array}\right]$ and $A=\left[\begin{array}{ll}A_{11} & A_{12} \\ A_{21} & A_{22}\end{array}\right]$.

The absence of impulses of the descriptor system (8) is equivalent to the nonsingularity of matrix $A_{22}$; see article [35].

Next, take $v\left(x_{1}\right)=x^{T} E^{T} P x \geq 0$, where matrix $P$ satisfies the equation (9) and (10) as well as the inequality $E^{T} P=P^{T} E \geq 0$. Then $\varphi(x)=-x^{T} C^{T} C x \leq 0$, and thus the conditions (i) and (ii) of Theorem 1 hold. To prove that condition (iii) also holds, consider the descriptor system (8) extended by the condition $\varphi(x)=0$, which appears equivalent to the equation $C x=0$. Since the pair $(A-s E, C)$ is finite mode detectable, by Theorem 1 we have that $x(t) \rightarrow 0$ when $t \rightarrow \infty$.

Example 2. Take the following nonlinear autonomous system

$$
\begin{aligned}
& \dot{x}=-x y^{2}, \dot{y}=e^{z}+z-1, \\
& e^{z}+z=y+1,
\end{aligned}
$$

with the equilibrium point $(x, y, z)=(0,0,0)$. Take the function $v(x)=0.5 x^{2}$. We find $\dot{\boldsymbol{v}}=\boldsymbol{\varphi}(x, y)=-x^{2} y^{2}$. Therefore, the conditions (i) and (ii) of Theorem 1 are satisfied. However, the condition (iii) is not satisfied. Indeed, the equation of the autonomous system plus the equation $\varphi(x, y)=-x^{2} y^{2}=0$ admit a solution $x(t)=0$ and $y(t)=e^{t} y_{0}$, so $y(t) \rightarrow \infty$ when $t \rightarrow \infty$.

It is easy to check that the true solution of the autonomous system is

$$
\begin{aligned}
& x(t)=e^{-0.5 y_{0}^{2}\left(e^{2 t}-1\right)} x_{0} \rightarrow 0(t \rightarrow \infty), \\
& y(t)=e^{t} y_{0} \rightarrow \infty(t \rightarrow \infty),
\end{aligned}
$$

and $z(t)$ is given by the implicit function (11).

Example 3. Consider the descriptor system given by

$$
\begin{aligned}
& \dot{x}=-x z, \dot{y}=z-y, \\
& z^{2}-x=0 .
\end{aligned}
$$


From the latter equation it follows that the consistent initial values $x(0)=x_{0}, y(0)=y_{0}$ and $z(0)=z_{0}$ have to satisfy $z_{0}^{2}-x_{0}=0$, and that $x(t) \geq 0$ for all $t$. The equation (13) has two solutions $z=-\sqrt{x}$ and $z=\sqrt{x}$. If we take the former solution, by solving the differential equations (12), we obtain

$$
x(t)=1 /\left(0.5 t+\left(z_{0}\right)^{-1}\right)^{2}, z(t)=1 /\left(0.5 t+\left(z_{0}\right)^{-1}\right),
$$

( $y(t)$ is not presented) therefore, a finite escape time appears ( at $t=-2\left(z_{0}\right)^{-1}$ ). In this case, we take $\mathbf{N}=\mathbf{N}_{1} \times \mathbf{N}_{2}$, where $\mathbf{N}_{1}=[0, \infty] \times[-\infty, \infty]$ and $\mathbf{N}_{2}=[-\infty, 0]$. If we take the solution $z=\sqrt{x}$, by solving the differential equation (12), we obtain formally the same solution (14) for $x(t)$ and $z(t)$, then

$$
y(t)=e^{-t} y_{0}+\int_{0}^{t} e^{s-t} /\left(0.5 s+\left(z_{0}\right)^{-1}\right) d s .
$$

Therefore, a finite escape time does not appear. In this case, we take $\mathbf{N}=\mathbf{N}_{1} \times \mathbf{N}_{2}$, where $\mathbf{N}_{1}$ is the same, and $\mathbf{N}_{2}=[0, \infty]$.

Moreover, we can apply Theorem 1 on the case $\mathbf{N}_{2}=[0, \infty]$. Take $\boldsymbol{v}(x)=0.5 x^{2} \geq 0$. Then $\dot{\boldsymbol{v}}=-z^{5} \leq 0$ in $\mathbf{N}$. The equation $\dot{\boldsymbol{v}}=0$ implies that $z=0$ and, by (13), $x=0$, while the solution of the right equation in (12) is $y=e^{-t} y_{0}$. The latter solution $(x, y, z)=\left(0, e^{-t} y_{0}, 0\right)$ satisfies the condition (iii) of Theorem 1 , therefore the solution (12) and (13) tend to the origin when $t \rightarrow \infty$ for all initial values, which is already seen by (14) and (15) (That $y(\infty)=0$ can be checked by applying the l'Hopital rule on (15)).

Corollary 1: Consider that the descriptor system (1) and (2) is given, such that Assumptions 1 and 2 hold, and let there exist a function $v\left(x_{1}\right)$ satisfying the condition (i) and (ii) of Theorem 1. If the system of algebraic equations $\boldsymbol{h}(x)=0$, $\dot{\boldsymbol{v}}=0, \ddot{\boldsymbol{v}}=0, \ldots$ has unique solution $x=0$, then the solution $x(t)$ of the descriptor system given by (1) and (2) satisfies $x(\infty)=\lim _{t \rightarrow \infty} x(t)=0$ for all initial conditions $x(0)=x_{0}$.

Proof. We have to prove only that the expressions $\ddot{v}=0, \ldots$ are actually algebraic equations in $x$. Consider the extended set of equations given by (1) and (2), and $\dot{\boldsymbol{v}}=\boldsymbol{\varphi}(x)=0$ too. By the differentiation of this equation in the variable $t$, we obtain the equation $\ddot{v}=0$. To find $\ddot{v}$, as a function of $x_{1}$ and $x_{2}$, we write at first

$$
\ddot{v}=\frac{\partial \varphi}{\partial x_{1}} \dot{x}_{1}+\frac{\partial \varphi}{\partial x_{2}} \dot{x}_{2},
$$


Since $\dot{x}_{2}$ is not known, we differentiate in $t$ the equation $\boldsymbol{h}(x)=0$ and obtain $\frac{\partial \boldsymbol{h}}{\partial x_{1}} \dot{x}_{1}+\frac{\partial \boldsymbol{h}}{\partial x_{2}} \dot{x}_{2}=0$, from where, by using Proposition 1, we obtain $\dot{x}_{2}=-\left(\frac{\partial \boldsymbol{h}}{\partial x_{2}}\right)^{-1} \frac{\partial \boldsymbol{h}}{\partial x_{1}} \boldsymbol{f}$.

Then by replacing this identity into (16), we obtain $\ddot{\boldsymbol{v}}=\left[\frac{\partial \boldsymbol{\varphi}}{\partial x_{1}}-\frac{\partial \boldsymbol{\varphi}}{\partial x_{2}}\left(\frac{\partial \boldsymbol{h}}{\partial x_{2}}\right)^{-1} \frac{\partial \boldsymbol{h}}{\partial x_{1}}\right] \boldsymbol{f}=0$.

The higher order time-derivatives of $v$, as function of $x_{1}$ and $x_{2}$, can be found in an obvious analogous way, which completes the proof.

Remark 5. If (1) and (2) is a given linear descriptor system (8), then Corollary 1 corresponds to the case when the pair $(A-s E, C)$ is finite mode observable, instead of solely finite mode detectable (like in Example 1).

In the next corollary, we consider that the vector $\boldsymbol{h}$ and $x_{2}$ in the system (1) and (2) are absent, i.e. we consider the autonomous system

$$
\dot{x}=\boldsymbol{f}(x), x \in \mathbf{N} \subseteq \mathbf{R}^{n},
$$

which satisfies $f(0)=0$ and $f$ has all-order partial derivatives.

The notion of locally weak observability for nonlinear systems is defined on page 733 of [36], and Theorem 3.1 of that paper gives the necessary and sufficient conditions. Here we adapt these results on the simpler autonomous (without control) system (17) the output of which is $y=\varphi(x)$. The operator $L_{f}$ is defined on scalar functions $\varphi(x)$ as $L_{f}(\varphi)=(\partial \varphi / \partial x) f$. Consider all possible scalar functions $L_{f}\left(\cdots L_{f}\left(\cdots L_{f}(\varphi) \cdots\right) \cdots\right)=: L_{f}^{k}(\varphi)$, where $k$ is the number of times the symbol $f$ appears. According to Theorem 3.1 of [36], the system is locally weakly observable at some $x \in \mathbf{N}$ if and only if the row-vectors $(\partial / \partial x) L_{f}^{k}(\varphi), k=0,1, \ldots$ have full rank $n$ at that $x$. This condition is related to the condition (iii) of the next corollary.

Corollary 2: Consider the autonomous system (17), having a unique solution without a finite escape time for all initial values $x_{0} \in \mathbf{N}$, and suppose that there exist a function $v(x)$ satisfying the conditions:

(i) $\boldsymbol{v}(x) \geq 0$ for all $x \in \mathbf{N}$, and $\boldsymbol{v}(0)=0$, 
(ii) $\varphi(x):=\frac{\partial \boldsymbol{v}}{\partial x} \boldsymbol{f}(x)=\dot{\boldsymbol{v}} \leq 0$ for all $x \in \mathbf{N}$

(iii) The unique solution of all algebraic equations $L_{f}^{k}(\varphi)=0, k=0,1, \ldots$ is $x=0$.

Then the solution $x(t)$ of the system (17) satisfies $x(\infty)=\lim _{t \rightarrow \infty} x(t)=0$ for all initial conditions $x(0)=x_{0}$.

Proof. It is a direct consequence of Corollary $1 .+$

\subsection{Feedback Stabilizability of Nonlinear Descriptor Systems}

Let us consider the following affine-in-control nonlinear descriptor system:

$$
\begin{aligned}
& \dot{x}_{1}=\boldsymbol{f}\left(x_{1}, x_{2}\right)+\boldsymbol{G}\left(x_{1}, x_{2}\right) u, \\
& 0=\boldsymbol{h}\left(x_{1}, x_{2}\right)+\boldsymbol{E}\left(x_{1}, x_{2}\right) u
\end{aligned}
$$

where $\quad x_{1} \in \mathbf{N}_{1} \subseteq \mathbf{R}^{n_{1}}, x_{2} \in \mathbf{N}_{1} \subseteq \mathbf{R}^{n_{2}}, \quad x=\left[\begin{array}{ll}x_{1} & x_{2}\end{array}\right]^{T} \in \mathbf{N} \subseteq \mathbf{R}^{n}, u=\left[u_{1} \cdots u_{m}\right]^{T}$, $u_{1}, \ldots, u_{m}$ are scalars, $\boldsymbol{G}=\left[\boldsymbol{g}_{1}, \ldots, \boldsymbol{g}_{m}\right], \boldsymbol{E}=\left[\boldsymbol{e}_{1}, \ldots, \boldsymbol{e}_{m}\right]$, and $\boldsymbol{g}_{1}, \ldots, \boldsymbol{g}_{m}, \boldsymbol{e}_{1}, \ldots, \boldsymbol{e}_{m}$ are column-vectors.

Theorem 2: Let there exist a function $v\left(x_{1}\right)$ such that

(i) $\boldsymbol{v}\left(x_{1}\right) \geq 0$ for all $x_{1} \in \mathbf{N}_{1}$, and $\boldsymbol{v}(0)=0$,

(ii) $\boldsymbol{\varphi}\left(x_{1}, x_{2}\right):=\frac{\partial \boldsymbol{v}}{\partial x_{1}} \boldsymbol{f}\left(x_{1}, x_{2}\right) \leq 0$ for all $x \in \mathbf{N}$ satisfying (2).

Introduce the row-vector function $\psi\left(x_{1}, x_{2}\right)$ by

$$
\boldsymbol{\psi}\left(x_{1}, x_{2}\right)=\frac{\partial \boldsymbol{v}}{\partial x_{1}} \boldsymbol{G}\left(x_{1}, x_{2}\right)
$$

We apply the control

$$
u=\boldsymbol{u}\left(x_{1}, x_{2}\right)=-\boldsymbol{Q}\left(x_{1}, x_{2}\right) \boldsymbol{\psi}\left(x_{1}, x_{2}\right)^{T},
$$

where $\boldsymbol{Q}\left(x_{1}, x_{2}\right)$ is an arbitrary symmetric positive definite matrix function in $\mathbf{N}$.

(iii) The solution $x(t)$ of the descriptor systems given by

$$
\begin{aligned}
& \dot{x}_{1}=\boldsymbol{f}\left(x_{1}, x_{2}\right), \\
& \boldsymbol{h}\left(x_{1}, x_{2}\right)=0, \quad \boldsymbol{\varphi}\left(x_{1}, x_{2}\right)=0, \quad \boldsymbol{\psi}\left(x_{1}, x_{2}\right)=0,
\end{aligned}
$$

satisfies $x(\infty)=\lim _{t \rightarrow \infty} x(t)=0$ for all initial conditions $x(0)=x_{0}$, 
Then, under Assumptions 1 and 2 for the closed-loop descriptor system given by (18), (19) and (20), the solution $x(t)$ of the closed-loop descriptor system satisfies $x(\infty)=\lim _{t \rightarrow \infty} x(t)=0$ for all initial condition $x(0)=x_{0}$.

Proof. In fact, this theorem is a consequence of Theorem 1. Indeed, having in mind the inequality

$$
\dot{\boldsymbol{v}}=\frac{\partial \boldsymbol{v}}{\partial x_{1}} \boldsymbol{f}+\frac{\partial \boldsymbol{v}}{\partial x_{1}} \boldsymbol{G} u=\frac{\partial \boldsymbol{v}}{\partial x_{1}} \boldsymbol{f}-\frac{\partial \boldsymbol{v}}{\partial x_{1}} \boldsymbol{G} \boldsymbol{Q}\left(\frac{\partial \boldsymbol{v}}{\partial x_{1}} \boldsymbol{G}\right)^{T} \leq 0,
$$

we can deduce that the identity $\dot{\boldsymbol{v}}=0$ is equivalent with the set of identities

$$
\frac{\partial \boldsymbol{v}}{\partial x_{1}} \boldsymbol{f}=0 \text { and } \frac{\partial \boldsymbol{v}}{\partial x_{1}} \boldsymbol{g}_{i}=0, i=1,2, \ldots, m
$$

and consequently $\psi\left(x_{1}, x_{2}\right)=0$ along the trajectories of the closed-loop system.

Remark 5. The matrix $\boldsymbol{Q}\left(x_{1}, x_{2}\right)$ has been introduced in order to enable achieving a proper balance between the usually opposite tasks:

(i) Reducing the magnitude of the control; and

(ii) Obtaining an acceptable distance to instability boundary of the closed-loop system, which in this paper for nonlinear descriptor systems is not defined rigorously; it is conditionally assumed based on Lurye's theory [3].

(Recall that for stable linear autonomous systems, $\dot{x}=A x$, the distance to instability is the smallest distance to the imaginary axis among all eigenvalues of the matrix $A$ ).

Remark 6. It is shown in [40] that in the case of linear descriptor system, using a preliminary feedback, we can obtain an impulse-free descriptor system, which is a property of a part of Assumption 2. Similar pre-feedback could be applied on the nonlinear descriptor system (18), (19).

As in Corollaries 1 and 2, here we find sufficient conditions which guarantee that Condition (iii) of Theorem 2 holds without integrating nonlinear differential equations and without solving nonlinear algebraic systems. Introduce the Jacobi brackets $\left[\boldsymbol{f}, \boldsymbol{g}_{i}\right]$ between the vector functions $\boldsymbol{f}$ and $\boldsymbol{g}_{i}$, i.e. vector fields, defined as:

$\left[\boldsymbol{f}, \boldsymbol{g}_{i}\right]=\frac{\partial \boldsymbol{g}_{i}}{\partial x_{1}} \boldsymbol{f}-\frac{\partial \boldsymbol{f}}{\partial x_{1}} \boldsymbol{g}_{i}$, and then define inductively the following operator ad: $\operatorname{ad}_{f}^{0} \boldsymbol{g}_{i}=\boldsymbol{g}_{i}, \quad \operatorname{ad}_{f}^{k+1} \boldsymbol{g}_{i}=\left[\boldsymbol{f}, \operatorname{ad}_{f}^{k} \boldsymbol{g}_{i}\right], \quad k=0,1,2, \ldots$ Further, let us denote by $\mathrm{L}_{x_{1}, x_{2}}\left(\boldsymbol{f}, \boldsymbol{g}_{1}, \ldots \boldsymbol{g}_{m}\right)$ the set of vector functions $\boldsymbol{f}$ and $\operatorname{ad}_{f}^{k} \boldsymbol{g}_{i}, i=1, \ldots, m$, $k=0,1, \ldots$ 
Corollary 3: Let there exist a function $v\left(x_{1}\right)$ such that

(i) $\boldsymbol{v}\left(x_{1}\right) \geq 0$ for all $x_{1} \in \mathbf{N}_{1}, \boldsymbol{v}(0)=0$,

(ii) $\boldsymbol{\varphi}\left(x_{1}, x_{2}\right):=\frac{\partial \boldsymbol{v}}{\partial x_{1}} \boldsymbol{f}\left(x_{1}, x_{2}\right)=0$ for all $x \in \mathbf{N}$ satisfying (2).

(iii) $\operatorname{rank}\left(\mathrm{L}_{x_{1}, x_{2}}\left(\boldsymbol{f}, \boldsymbol{g}_{1}, \ldots \boldsymbol{g}_{m}\right)\right)=n_{1}$, for all nonzero $x \in \mathbf{N}$ satisfying (22),

(iv) the algebraic equations given by $\frac{\partial v}{\partial x_{1}}=0$ and (22) have unique solution $x_{1}=0, x_{2}=0$.

With application of the chosen control (20), and under Assumptions 1 and 2 for the closed-loop descriptor system described by means of (18), (19) and (20), the solution $x(t)$ of closed-loop descriptor system satisfies $x(\infty)=\lim _{t \rightarrow \infty} x(t)=0$ for all initial conditions $x(0)=x_{0}$.

Proof. A consequence of the identities (23) and $\dot{x}_{1}=\boldsymbol{f}\left(x_{1}, x_{2}\right)$ is the following identity

$$
\begin{gathered}
0=\frac{d}{d t}\left(\frac{\partial \boldsymbol{v}\left(x_{1}(t)\right)}{\partial x_{1}} \boldsymbol{g}_{i}\left(x_{1}(t), x_{2}(t)\right)\right)=\frac{\partial \boldsymbol{v}}{\partial x_{1}} \frac{\partial \boldsymbol{g}_{i}}{\partial x_{1}} \boldsymbol{f}+\boldsymbol{g}_{i}^{\mathrm{T}} \frac{\partial^{2} \boldsymbol{v}}{\partial x_{1}^{2}} \boldsymbol{f} \\
=\frac{\partial \boldsymbol{v}}{\partial x_{1}}\left(\left[\boldsymbol{f}, \boldsymbol{g}_{i}\right]+\frac{\partial \boldsymbol{f}}{\partial x_{1}} \boldsymbol{g}_{i}\right)+\boldsymbol{f}^{\mathrm{T}} \frac{\partial^{2} \boldsymbol{v}}{\partial x_{1}^{2}} \boldsymbol{g}_{i}=\frac{\partial \boldsymbol{v}}{\partial x_{1}}\left[\boldsymbol{f}, \boldsymbol{g}_{i}\right]+\left(\frac{\partial \boldsymbol{v}}{\partial x_{1}} \frac{\partial \boldsymbol{f}}{\partial x_{1}}+\boldsymbol{f}^{\mathrm{T}} \frac{\partial^{2} \boldsymbol{v}}{\partial x_{1}^{2}}\right) \boldsymbol{g}_{i} \\
=\frac{\partial \boldsymbol{v}}{\partial x_{1}}\left[\boldsymbol{f}, \boldsymbol{g}_{i}\right]+\frac{\partial}{\partial x_{1}}\left(\frac{\partial \boldsymbol{v}}{\partial x_{1}} \boldsymbol{f}\right) \boldsymbol{g}_{i}=\frac{\partial \boldsymbol{v}}{\partial x_{1}}\left[\boldsymbol{f}, \boldsymbol{g}_{i}\right]
\end{gathered}
$$

where the property that matrix $\frac{\partial^{2} v}{\partial x_{1}^{2}}$ is symmetric is used, which is true due to the equality of mixed second-order partial derivatives. By means of mathematical induction, we obtain the identity $\frac{\partial \boldsymbol{v}}{\partial x_{1}} \operatorname{ad}_{f}^{k} \boldsymbol{g}_{i}=0, i=1, \ldots, m, k=0,1, \ldots$.

Then by the condition (iii) of Corollary 3 and by $\frac{\partial \boldsymbol{v}}{\partial x_{1}} \mathrm{~L}_{x_{1}, x_{2}}\left(\boldsymbol{f}, \boldsymbol{g}_{1}, \ldots \boldsymbol{g}_{m}\right)=0$, we obtain $\frac{\partial \boldsymbol{v}}{\partial x_{1}}=0$, for all $x \in \mathbf{N}$ except $x=0$. Furthermore due to condition (iv), we have $x_{1}=0$ and $x_{2}=0$. + 
In the subsequent corollary, we consider that the equation (19) is void and consequently the vector $x_{2}$ is void too. That is, we consider the affine-in-control system

$$
\dot{x}=\boldsymbol{f}(x)+\boldsymbol{G}(x) u, x \in \mathbf{N} \subseteq \mathbf{R}^{n}, u \in \mathbf{R}^{m},
$$

which satisfies $\boldsymbol{f}(0)=0$ and the functions $\boldsymbol{f}$ and $\boldsymbol{G}$ have partial derivatives of all orders. In this case, the definition of set $\mathrm{L}_{x}\left(\boldsymbol{f}, \boldsymbol{g}_{1}, \ldots, \boldsymbol{g}_{m}\right)$ is obvious. By Theorem 2.2 of [36], if rank of $\mathrm{L}_{x}\left(\boldsymbol{f}, \boldsymbol{g}_{1}, \ldots, \boldsymbol{g}_{m}\right)$ is full at some $x \in \mathbf{N} \subseteq \mathbf{R}^{n}$ (equal to $n$ ), then the system (24) is locally weakly controllable at $x$.

Corollary 4: Let there exist a function $v(x)$ such that

(i) $\boldsymbol{v}(x) \geq 0$ for all $x \in \mathbf{N}$, and $\boldsymbol{v}(0)=0$,

(ii) $\varphi(x):=\frac{\partial \boldsymbol{v}}{\partial x} \boldsymbol{f}(x)=0$ for all $x \in \mathbf{N}$.

(iii) $\operatorname{rank}\left(\mathrm{L}_{x}\left(\boldsymbol{f}, \boldsymbol{g}_{1}, \ldots, \boldsymbol{g}_{m}\right)\right)=n$ for all nonzero $x \in \mathbf{N}$ satisfying $\boldsymbol{\varphi}(x)=0$ and $\psi(x)=0$,

(iv) The unique solution of the algebraic equations given by $\frac{\partial \boldsymbol{v}}{\partial x}=0, \varphi(x)=0$ and $\boldsymbol{\psi}(x)=0$, is $x=0$.

We apply the following control law

$$
\boldsymbol{u}(x)=-\boldsymbol{Q}(x)\left(\frac{\partial \boldsymbol{v}}{\partial x} \boldsymbol{G}\right)^{T},
$$

where $\boldsymbol{Q}(x)$ is an arbitrary symmetric positive definite matrix function in $\mathbf{N}$. If the closed loop system of (24) and (25) has a unique solution without finite escape times, then the solution $x(t)$ of the closed-loop system satisfies $x(\infty)=\lim _{t \rightarrow \infty} x(t)=0$ for all initial conditions $x(0)=x_{0}$.

Proof. It is a direct consequence of Corollary 3.

\section{Application to Quadcopter Stabilization Problem}

Firstly, an analysis of quadcopters flight dynamics is presented from the viewpoint of control. Thereafter the respective results of the simulation experiments and relevant findings are discussed. 


\subsection{Feedback Stabilizability of Nonlinear Descriptor Systems}

The nonlinear dynamic model of the quadcopter consists the following set of differential equations [39]:

$$
\left.\begin{array}{ll}
\dot{x}=v_{x}, \quad \dot{y}=v_{y}, \quad \dot{z}=v_{z}, & \dot{\phi}=\omega_{\phi}, \quad \dot{\theta}=\omega_{\theta}, \quad \dot{\psi}=\omega_{\psi}, \\
\dot{v}_{x}=m^{-1}[\cos \phi \cdot \sin \theta \cdot \cos \psi+\sin \phi \cdot \sin \psi] \cdot U_{1}, & \dot{p}=J_{x x}^{-1}\left(J_{y y}-J_{z z}\right) q r+J_{x x}^{-1} \cdot U_{2}, \\
\dot{v}_{y}=m^{-1}[\cos \phi \cdot \sin \theta \cdot \sin \psi-\sin \phi \cdot \cos \psi] \cdot U_{1}, & \dot{q}=J_{y y}^{-1}\left(J_{z z}-J_{x x}\right) p r+J_{y y}^{-1} \cdot U_{3}, \\
\dot{v}_{z}=-g+m^{-1}[\cos \phi \cdot \cos \theta] \cdot U_{1}, & \dot{r}=J_{z z}^{-1}\left(J_{x x}-J_{y y}\right) p q+J_{z z}^{-1} \cdot U_{4} \cdot
\end{array}\right\}
$$

In here, $U_{1}, U_{2}, U_{3}, U_{4}$ are transformed angular velocities of the four propellers, variables $x, y, z$ and $\phi, \theta, \psi$ represent linear and angular displacements and their derivatives $v_{x}, v_{y}, v_{z}$, respectively $\omega_{\phi}, \omega_{\theta}, \omega_{\psi}$, are all with respect to the Earth's coordinate frame. On the other hand, $p, q, r$ denote angular rates with respect to the frame of flying body. The parameters $m, g$ denote total mass and gravitational acceleration in the negative $z$-axis, and $J_{x x}, J_{y y}, J_{z z}$ are the moments of inertia with respect to $x-y-z$ axes. In addition, the following three algebraic equations

$$
\begin{aligned}
& \omega_{\phi}=p+s_{\phi} t_{\theta} \cdot q+c_{\phi} t_{\theta} \cdot r, \\
& \omega_{\theta}=c_{\phi} \cdot q-s_{\phi} \cdot r, \\
& \omega_{\psi}=s_{\phi} / c_{\theta} \cdot q+c_{\phi} / c_{\theta} \cdot r,
\end{aligned}
$$

where $c_{(.)}, s_{(.)}$and $t_{(.)}$is a short notation for cos-, sin- and tan- functions, respectively, complete the representation model.

In general, the equations (26) and (27) together describe the nonlinear descriptor system for quadcopters. Actually, the differential equation (26) corresponds to the differential equation (18) while the algebraic equations (27) correspond to the algebraic equation (19).

Indeed, it is of the great importance how to select or construct a particular Lyapunov-like function for this particular problem. In order to find Lyapunov-like function for the system without control, i.e. for $\dot{x}_{1}=\boldsymbol{f}\left(x_{1}, x_{2}\right)$, consider that all inputs in (26) are zero, that is. $U_{1}=U_{2}=U_{3}=U_{4}=0$. Thus, we obtain:

$$
\left.\begin{array}{llll}
\dot{x}=v_{x}, & \dot{v}_{x}=0, & \dot{\phi}=\omega_{\phi}, & \dot{p}=J_{x x}^{-1}\left(J_{y y}-J_{z z}\right) q r \\
\dot{y}=v_{y}, & \dot{v}_{y}=0, & \dot{\theta}=\omega_{\theta}, & \dot{q}=J_{y y}^{-1}\left(J_{z z}-J_{x x}\right) p r \\
\dot{z}=v_{z}, & \dot{v}_{z}=-g, & \dot{\psi}=\omega_{\psi}, & \dot{r}=J_{z z}^{-1}\left(J_{x x}-J_{y y}\right) p q .
\end{array}\right\}
$$

It is well-known that the Lyapunov stability theory is in fact energy-based methodology. The quadcopter is a physical system and furthermore, it is an energy 
conservative system [13]. In other words, the sum of the kinetic and potential energy does not change in time. Therefore, the Lyapunov-like function, $v$ in our case, is defined as $\boldsymbol{v}=\alpha \boldsymbol{e}_{t}+\beta \boldsymbol{e}_{r}$ with some dimensionless tuning coefficients $\alpha$ and $\beta$. Furthermore, due to the physics of quadcopter, formula $\boldsymbol{e}_{t}=0.5 m\left(v_{x}^{2}+v_{y}^{2}+v_{z}^{2}+2 g z\right)$ describes the translational energy of motion while formula $\boldsymbol{e}_{r}=0.5\left(J_{x x} p^{2}+J_{y y} q^{2}+J_{z z} r^{2}\right)$ describes the rotational energy. Therefore

$$
\boldsymbol{v}=0.5 \alpha m\left(v_{x}^{2}+v_{y}^{2}+v_{z}^{2}+2 g z\right)+0.5 \beta\left(J_{x x} p^{2}+J_{y y} q^{2}+J_{z z} r^{2}\right)
$$

does represent the total energy in the considered plant system. In turn, for $\dot{\boldsymbol{v}}$ we have:

$$
\dot{\boldsymbol{v}}=\alpha m\left(v_{x} \dot{v}_{x}+v_{y} \dot{v}_{y}+v_{z} \dot{v}_{z}+g \dot{z}\right)+\beta\left(J_{x x} p \dot{p}+J_{y y} q \dot{q}+J_{z z} r \dot{r}\right) .
$$

Then by substituting equation (28), one can obtain $\dot{\boldsymbol{v}}=0$. Consequently, equations (29) and (30) satisfy conditions (i), (ii) of Theorem 2 or else the Corollary 3. For the quadcopter descriptor representation (26) and (27), the selected Lyapunov-like function is shown to be useful for obtaining the needed control vector. Formula (20) along with $Q_{1}\left(x_{1}, x_{2}\right)=I_{4}$ gives the following stabilizing control set $u_{1}, u_{2}, u_{3}, u_{4}$ :

$$
\begin{aligned}
& u_{1}=-\alpha\left[v_{x}\left(c_{\phi} s_{\theta} c_{\psi}+s_{\phi} s_{\psi}\right)+v_{y}\left(c_{\phi} s_{\theta} s_{\psi}-s_{\phi} c_{\psi}\right)+v_{z}\left(c_{\phi} c_{\theta}\right)\right] \\
& u_{2}=-\beta p=-\beta\left(\omega_{\phi}-\omega_{\psi} s_{\theta}\right) \\
& u_{3}=-\beta q=-\beta\left(\omega_{\theta} c_{\phi}-\omega_{\psi} s_{\phi} c_{\theta}\right) \\
& u_{4}=-\beta r=-\beta\left(\omega_{\psi} c_{\phi} c_{\theta}-\omega_{\theta} s_{\phi}\right)
\end{aligned}
$$

The implementation of feedback nonlinear stabilizing control, which is actually an inner loop of quadcopter stabilization, has been achieved by attaching $u_{i}$ to the tracking control $u_{t c i}$.

$U_{i}=\gamma \cdot u_{t c i}+u_{i}$

where: $i \in\{1, \cdots, 4\}, \gamma$ is a dimensionless tuning parameter, and $U_{i}$ represent the actually applied control signals to quadcopter's nonlinear descriptor system. The intended effect of the inner and outer loop is to provide for faster stabilization and slower trajectory tracking, respectively.

\subsection{Simulation Experiments of the Quadcopter Stabilization}

The theory of the paper has been tested on quadcopter stabilization, under the task of reaching the desired point in space $[x, y, z]^{T}=[5,5,10]^{T}$ while keeping the quadcopter head (yaw angle) fixed, i.e. $\psi=0$. The initial posture is taken 
$[0,0,0]^{T}$ and $\psi=0$. The comparison on the quadcopter stabilization is conducted on four different simulations. The control magnitude between the tracking control and stabilizing control in (32) has been achieved by tuning parameters $\alpha, \beta, \gamma$ presented on Table 1.

Table 1

Control magnitude tuning parameters

\begin{tabular}{|c|c|c|c|}
\hline Simulation number & $\alpha$ & $\beta$ & $\gamma$ \\
\hline 1 & 0 & 0 & 1 \\
\hline 2 & 0.08 & 0.01 & 1 \\
\hline 3 & 0.42 & 0.02 & 0.9 \\
\hline 4 & 0.83 & 0.03 & 0.8 \\
\hline
\end{tabular}

The first simulation ('1') elaborates the stabilizing achieved only from the tracking control. Then in the following simulations, we gradually decrease the control magnitude of the tracking control (i.e. the parameter $\gamma$ ) and simultaneously increase control magnitude of the stabilizing feedback control (31) (i.e. the parameters $\alpha$ and $\beta$ ).

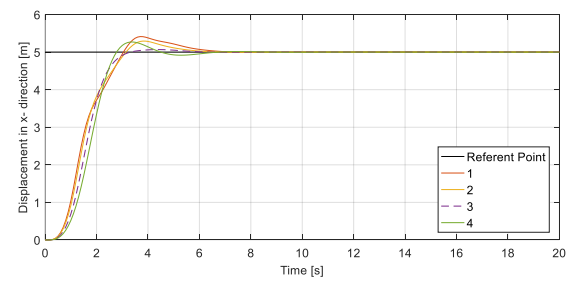

Figure 2

Linear displacement in the $\mathrm{x}$-axis

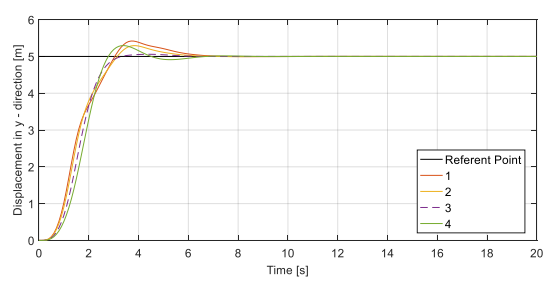

Figure 4

Linear Displacement in the $y$-axis

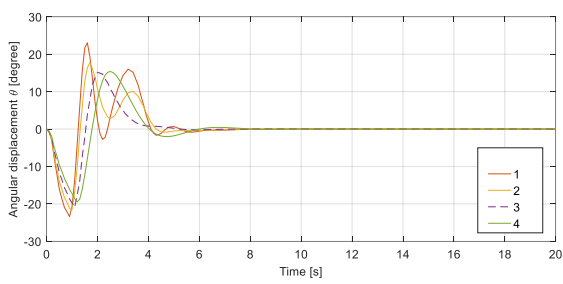

Figure 3

Angular displacement $\phi$

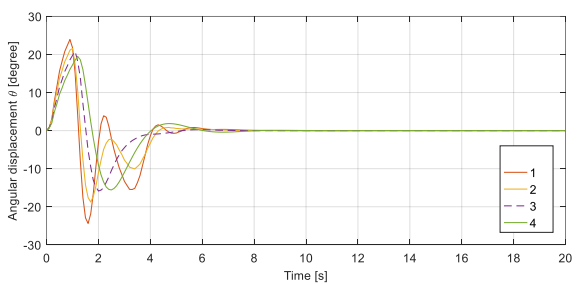

Figure 5

Angular displacement $\theta$ 


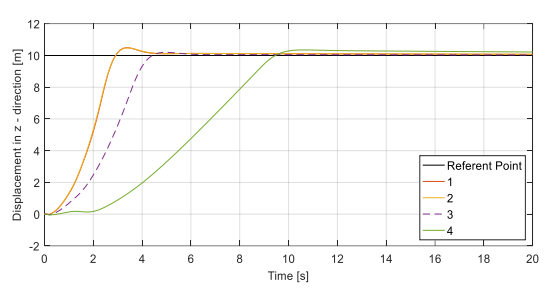

Figure 6

Linear Displacement in the z-axis

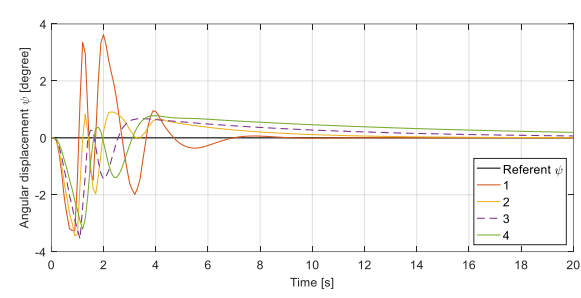

Figure 7

Angular displacement $\psi$

In comparison with other simulations, in particular with simulation number 1 (pure tracking control), simulation number 3 results on achieving a better tracking of quadcopter in $x, y$ direction, while the height control ( $z$-direction) it increases the rising time, but the settling time remains almost the same. By analyzing Figures 3, 5 and 7, the comparison between a pure tracking control stabilization and the mixed stabilization control (the simulation number 3 ) shows that the latter one improves the stabilization of the quadcopter by reducing orientation chattering.

\section{Conclusions}

In this paper, in addition to designing the nonlinear stabilizing control based on Lyapunov-like stability for nonlinear descriptor system, a new approach for representing the quadcopter, as a nonlinear descriptor system, has been elaborated. It is presented and demonstrated through numerical simulation, the application of the new approach on quadcopter stabilization.

The presented numerical results show that the stabilization of the quadcopter has been improved by introducing a mixed stabilization control (tracking control + stabilizing control derived from Lyapunov-like stability conditions). By decreasing the control magnitude of the tracking control and simultaneously increasing the control magnitude of the stabilization control, a larger converging time appears, which appears to be the only drawback of this new methodology.

Future theoretical extension of the presented results is foreseen, for the further enhancement of the system efficiency as argued in [37]. The other direction of future research will be focused on the improvement of the stabilization of the quadcopter towards a faster convergence and on the application toward other flying objects [38]. We would also like to examine the actual implementation of the stabilization control in a "real-world-scale" quadcopter. This is an open challenge, and it depends on future investment.

\section{References}

[1] B. Brogliato, R. Lozano, B. Maschke, O. Egeland: Dissipative Systems Analysis and Control Theory and Application, Springer, London, UK, 2007 
[2] P. D. Franco, G. Scarciotti, A. Astolfi: A note on the stability of nonlinear differential-algebraic systems, IFAC PapersOnLine, Vol. 50(1), 2017, pp. $7421-7426$

[3] A. I. Lurye: Certain Nonlinear Problems in the Theory of Automatic Control (in Russian, Nekatorie nelineynie zadachi teorii avtomaticheskogo regulirovaniya). Moskva, RU-USSR: Gostehizdat, 1951

[4] J. P. La Salle, S. Lefschetz: Stability by Liapunov's Direct Method with Applications. New York, NY - USA: Academic Press, 1961

[5] T. Watada, M. Ikeda, Y. Ohta, D. D. Siljak: Parametric absolute stability of multivariable Lurye systems, Automatica, Vol. 36, 2000, pp. 1365-1372

[6] T. Watada, M. Ikeda: Absolute stability criteria for multivariable Lurye systems with asymmetric nonlinearities, Proceedings of the $10^{\text {th }}$ IFAC/IFORS/IMACS/IFIP Symposium on Large Scale Systems Theory and App, Oxford, UK: Pegamon Elsevier Science, 2004, Vol. 2, pp. 475481

[7] T. Watada, M. Ikeda, E. Uezato: Stability theory for descriptor systems with non-smooth nonlinearities, Proceeding of the $17^{\text {th }}$ Int. Symposium on Mathematical Theory of Networks and Systems, 2006, pp. 1626-1631

[8] T. Watada, M. Ikeda, E. Uezato: Absolute stability of multivariable luryetype descriptor systems, Proceeding of the $17^{\text {th }}$ IFAC World Congress, Seoul, R. Korea, July 2008, pp. 6021-6026

[9] C. I. Byrnes, A. Isidori, J. C. Willems: Passivity, feedback equivalence, and the global stabilization of minimum phase nonlinear systems, IEEE Transaction on Automatic Control, Vol. 36(11), 1991, pp. 1228-1240

[10] A. Fradkov: Passification of non-square linear systems and feedback Yakubovich Kalman-Popov lemma, European Journal of Control, Vol. 9(6), 2003, pp. 577-586

[11] A. Saberi, P. V. Kokotovic, H. J. Sussmann: Global stabilization of partially linear composite systems, SIAM Journal of Control and Optimization, Vol. 28(6), 1990, pp. 1491-1503

[12] C. Yang, J. Sun, Q. Zhang, X. Ma: Lyapunov Stability and Strong Passivity Analysis for Nonlinear Descriptor Systems, IEEE Transactions on Circuits and Systems, Vol. 60(4), 2013, pp. 1003-1012

[13] J. D. Stefanovski: Infinite-dimensional conservative systems and a conservativity condition for the Saint-Venant equations, International Journal of Control, Vol. 73(13), 2000, pp. 1224-1234

[14] J. C. Willems: Dissipative dynamical systems, Part I: General Theory, Archive of Rational Mechanics Analysis, Vol. 45(5), 1972, pp. 321-352 
[15] J. C. Willems: Dissipative dynamical systems, Part II: Linear systems with quadratic supply rates, Archive of Rational Mechanics Analysis, Vol. 45(5), 1972, pp. 352-393

[16] D. J. Hill, P. J. Moylan: Dissipative dynamical systems: Basic input output and state properties, Journal of the Franklin Institute, Vol. 309(5), 1980, pp. 327-357

[17] P. C. Muller: Modelling and control of mechatronic systems by the descriptor approach, Journal of Theoretical and Applied Mechanics, Vol. 43(3), 2005, pp. 593-607

[18] A. Iggidr, B. Kalitine, R. Outbib: Semidefinite Lyapunov functions: Stability and stabilization, Math. of Cont., Sig., and Sys., Vol. 9(2), 1996, pp. $95-106$

[19] M. Larsen, M. Jankovic, P. V. Kokotovic: Coordinated passivation designs, Automatica, Vol. 39(2), 2003, pp. 335-341

[20] H. K. Khalil: Nonlinear Systems, Prentice Hall, Upper Saddle River, NJ, 2002, Ch. 6 Passivity

[21] Y. Yin, J. Stefanovski, G. Dimirovski, J. Zhao: Quadratic stability results for switched singular descriptor systems, Proceedings of the $9^{\text {th }}$ European Control Conference ECC07, Island of Kos, Greece, July 2007, pp. 696-701

[22] L. Y. Sun, J. Zhao, G. M. Dimirovski: Adaptive coordinated passivation control for generator excitation and thyristor-controlled series compensation system, Control Engineering Practice, Vol. 17(7), 2009, pp. $1295-1312$

[23] J. Zhao, D. J. Hill: Dissipativity theory for switched systems, IEEE Transactions on Automatic Control, Vol. 53(4), 2008, pp. 941-953

[24] Y. Liu, G. S. Stojanovski, M. J. Stankovski, G. M. Dimirovski, J. Zhao: Feedback passivation of switched nonlinear systems using storage-like functions, Int. Journal of Con. Auto. and Sys., Vol. 9(5), 2011, pp. 980-986

[25] D. Ma, G. M. Dimirovski: Passivity-based Switching Rule and Control Law Co-Design of Networked Switched Systems with Feedback Delays, in: G. M. Dimirovski, Editor, Complex Systems: Studies in Systems, Decision and Control, Cham, Springer, 2016, Vol. 55, pp. 249-266

[26] O. Araar, N. Aouf: Full linear control of a quadrotor UAV, LQ vs $H_{\infty}$, Proceedings of the UKACC International Conference on Control, Loughborough, UK, July 2014, pp. 133-138

[27] P. Wang, Z. Man, Z. Cao, J. Zheng, Y. Zhao: Dynamics modelling and linear control of quadcopter, Proceedings of the 2016 International Conference on Advanced Mechatronic Systems (ICAMechS), Melbourne, VIC, Australia, November 2016, pp. 498-503 
[28] S. Deskovski, V. Sazdovski, Z. Gacovski: Guidance Laws and Navigation Systems for Quadrotor UAV: Theoretical and Practical Findings, in: G. M. Dimirovski, Editor, Complex Systems: Studies in Systems, Decision and Control, Cham, Springer, 2016, Vol. 55, pp. 381-390

[29] Q. Quan: Introduction to Multicopter Design and Control. Singapore, SG: Springer Singapore, 2017

[30] S. K. Phang, S. Lai, F. Wang, M. Lan, B. M. Chen: Systems design and implementation with jerk-optimized trajectory generation for UAV calligraphy, Mechatronics, Vol. 30, 2015, pp. 65-75

[31] D. Bunjaku, J. D. Stefanovski, G. M. Dimirovski: Regularity conditions for control problem with descriptor systems, Proceedings of the $14^{\text {th }}$ IEEE International Conference on Control \& Automation (ICCA), Anchorage, AK-USA, June 2018, pp. 130-135

[32] S. L. Dai, J. Zhao, G. M. Dimirovski: A descriptor system approach to robust $H_{\infty}$ control for linear systems with time-varying uncertainties, Int. Journal of Systems Science, Vol. 40(12), 2009, pp. 1293-1306

[33] H. J. Sussmann, P. V. Kokotovic: The peaking phenomenon and the global stabilization of nonlinear systems, IEEE Transactions on Automatic Control, Vol. 36(4), 1991, pp. 424-440

[34] K. Takaba, N. Morihira, T. Katayama: A generalized Lyapunov theorem for descriptor system, Sys. and Con. Letters, Vol. 24(1), 1995, pp. 49-51

[35] J. Y. Ishihara, M. H. Terra: Impulse controllability and observability of rectangular descriptor systems, IEEE Transactions on Automatic Control, Vol. 46(6), 2001, pp. 991-994

[36] R. Hermann, A. J. Krener: Nonlinear controllability and observability, IEEE Trans. on Automatic Control, Vol. AC-22(5), 1977, pp. 728-740

[37] P. Kokotovic, M. Arcak: Constructive nonlinear control: A historical perspective, Automatica, Vol. 37(5), 2001, pp. 637-662

[38] B. L. Stevens, F. L. Lewis, E. N. Johnson: Aircraft control and simulation, Third Edition Dynamics, Controls Design, and Autonomous Systems, John Wiley \& Sons, Inc., Hoboken, New Jersey, 2016

[39] D. Bunjaku, G. Nadzinski, M. Stankovski, J. D. Stefanovski: Dynamic Modeling and Flight Control Design for Multicopter, International Review of Aerospace Engineering, Vol. 11(5), 2018, pp. 224-235

[40] D. Bunjaku, J. D. Stefanovski, G. M. Dimirovski, D. Juričić: New Approach on Solving Control Problems with Descriptor Systems, Journal of The Franklin Institute, Vol. 356(6), 2019, pp. 3270-3289

[41] G. Lu, D.W. Ho and L. Zhou: A note on the existence of a solution and stability for Lipschitz discrete-time descriptor systems, Automatica, Vol. 47(7), 2011, pp. 1525-1529 